\title{
Ionic model for highly compressed solid hydrogen
}

\author{
E.S. Yakub \\ Cybernetics Dept., Odessa National Economic University \\ 8 Preobrazhenskaya Str., Odessa 65082, Ukraine \\ E-mail: yakub@oneu.edu.ua
}

Received October 29, 2012

\begin{abstract}
We propose a simple ionic model for high-pressure conducting phase IV of solid hydrogen observed recently at room temperature. It is based on an assumption of dissociative ionization of hydrogen molecules $3 \mathrm{H}_{2}=2 \mathrm{H}_{2}^{(+)}+2 \mathrm{H}^{(-)}$induced by high compression. The model proposed predicts the first order transition of molecular hydrogen solid into partly ionic conducting phase at megabar pressures and describes the temperature dependence of resistivity at room temperature. Its predictions are consistent with high temperature shockcompression experiments which exhibit conductivity of multiply shocked hydrogen. Location of phase transition line, change of volume, and ionization degree in solid phase IV are estimated.
\end{abstract}

PACS: $05.70 . \mathrm{Ce}$ Thermodynamic functions and equations of state;

67.80.F- Solids of hydrogen and isotopes;

67.63.Cd Molecular hydrogen and isotopes;

64.60.Ej Studies/theory of phase transitions of specific substances.

Keywords: solid hydrogen, ionization, phase transition, conductivity, molecular dynamics.

\section{Introduction}

The quest for metallic hydrogen has begun many years ago just after publication of well-known theoretical work of Wigner and Huntington [1] and a lot of progress in experimental, theoretical and simulation work has been achieved. Electrical conductivity was observed both in static (see [2] for references) and dynamic [3] experiments but the rigorous proof of metallization still has not been found.

Recent diamond anvil cell (DAC) experiments [4] at room temperature and megabar pressures reveal unusual behavior of highly compressed solid hydrogen. According to Eremets and Troyan [4], solid hydrogen transforms first at $230 \mathrm{GPa}$ into a nonmetallic conducting phase, which exists up to $270 \mathrm{GPa}$. This phase is characterized by a low and rising with temperature conductivity, which is not typical for metals. Metallic hydrogen occur presumably (see discussion if Ref. 2) at higher pressures after the second phase transition [4].

The aim of this work is an attempt to understand the nature of this intermediate conducting nonmetallic phase (phase IV) of highly compressed hydrogen, discovered by Eremets and Troyan [4] at room temperature. We propose a simple model which explains anomalous properties of this nonmetallic phase by pressure induced ionization of $\mathrm{H}_{2}$ molecules. According to our estimations, first order phase transition into partly ionized state may occur at certain density and temperature when the energy needed for ionization of $\mathrm{H}_{2}$ molecule is compensated by the sum of the Coulomb attraction and polarization interaction of emerging $\mathrm{H}_{2}^{(+)}$and $\mathrm{H}^{(-)}$ions.

Parameters of ionic model, pressure, energy, and conductivity of partly ionic solid hydrogen are calculated directly using molecular dynamics technique and results are compared with the recent DAC experiments.

The ionic model is introduced in the next section. In Sec. 3 we describe the details of the underlying potential model, determine parameters of atom-atom, ion-ion and ion-atom interactions, and analyze the dependence of the Helmholtz free energy of highly compressed hydrogen solid on ionization degree. Properties of conducting phase estimated in molecular dynamics simulation are presented in Sec. 4. The results obtained and problems remaining are discussed in the last section.

\section{Partly ionic model for solid hydrogen}

Relatively low and slowly rising with temperature conductivity of solid hydrogen, observed in DAC experiments of Eremets and Troyan [4] is not typical for a metal but is quite usual in electrolytes. This rather trivial statement prompted us to check to what degree the simplest version 
of an ionic model is able to explain the observed behavior of highly compressed hydrogen solid.

Ionization of molecular hydrogen may correspond to the following reaction:

$$
3 \mathrm{H}_{2} \rightleftarrows 2 \mathrm{H}_{2}^{(+)}+2 \mathrm{H}^{(-)}
$$

Energetics of this reaction in ideal-gas state can be easily estimated using well-known constants [5]: electron affinity $(0.75 \mathrm{eV})$ of hydrogen atom, ionization potential $(15.61 \mathrm{eV})$ and dissociation energy $(4.75 \mathrm{eV})$ of $\mathrm{H}_{2}$ molecule. The reaction enthalpy $\left(\Delta I=11.48 \mathrm{eV}\right.$ per $\mathrm{H}_{2}$ molecule) is rather high and equilibrium ionization degree is negligible at room temperatures and atmospheric pressure. Nevertheless it can become noticeable at high temperatures or extreme compressions.

Energy needed for ionization of $\mathrm{H}_{2}$ molecule in condensed phase is essentially reduced by the Coulombic attraction of emerging $\mathrm{H}_{2}^{(+)}$and $\mathrm{H}^{(-)}$ions and their polarization interaction with surrounding $\mathrm{H}_{2}$ molecules. At high compression this effect may compensate the energy loss due to ionization and transition to the partly ionized state may occur.

To check this possibility quantitatively, we apply the following equation for Helmholtz free energy of partly ionic solid [6]:

$$
F(T, \rho, \xi)=F_{\text {lat }}^{(\mathrm{id})}(T, \rho, \xi)-k T \sum_{k} N^{(k)} \ln \left(\frac{v_{f}^{(k)}}{v}\right)
$$

Here $F_{\text {lat }}^{(\mathrm{id})}(T, \rho, \xi)$ is the free energy of ideal lattice gas, $T$ is temperature, $N^{k}=\left\{N^{(0)}, N^{(+)}, N^{(-)}\right\}, N^{0}$ is number of atoms in neutral $\mathrm{H}_{2}$ molecules, $N^{(+)}$is number of positive and $N^{(-)}$is number of negative atomic ions in volume $V$ (here and below we treat the $\mathrm{H}_{2}^{(+)}$molecular ion as a diatomic composed from two positive ions having formal charge $+0.5|e|)$; ) $N=N^{(-)}+N^{(+)}+N^{(0)}$ is the total number of protons, $v=V / N$ is specific volume (per one proton), $\rho=N / 2 V$ is molar density, $\xi$ is ionization degree, and $v_{f}^{(0)}, v_{f}^{(+)}, v_{f}^{(-)}$are, respectively, atomic free volume of a neutral atom in $\mathrm{H}_{2}$ molecule, of a positive ion in $\mathrm{H}_{2}^{(+)}$molecular ion, and of a negative atomic ion $\mathrm{H}^{(-)}$.

The degree of ionization $\xi$, when expressed in terms of $N^{k}$ is as follows:

$$
\xi=\left(N^{(-)}+N^{(+)}\right) /\left(N^{(0)}+N^{(-)}+N^{(+)}\right) .
$$

Within harmonic approximation, free volumes $v_{f}^{(0)}$, $v_{f}^{(+)}, v_{f}^{(-)}$, in turn, could be expressed through elastic constants $\alpha^{(0)}, \alpha^{(+)}, \alpha^{(-)}$of atomic (ionic) vibrations [6]:

$$
v_{f}^{(k)} \approx\left(\frac{\pi k T}{\alpha^{(k)}}\right)^{3 / 2}
$$

Finally, substituting the expression for ideal lattice gas contribution $F_{\text {lat }}^{(\mathrm{id})}(T, \rho, \xi)$ [6], one can write the final expression for Helmholtz free energy $F^{(\mathrm{id})}(T, \rho, \xi)$ as

$$
\begin{aligned}
F(T, \rho, \xi)=U_{N}^{(\mathrm{st})} & (\rho, \xi)+N \xi \Delta I+\xi N k T \ln \left(\frac{\alpha_{0}^{3}}{\alpha^{(-)} \alpha^{(+) 2}}\right)+ \\
& +N k T\left((1-\xi) \ln (1-\xi)+\frac{4}{3} \xi \ln \xi\right) .
\end{aligned}
$$

Here $U_{N}^{(\mathrm{st})}(\rho, \xi)$ is the static potential energy of partly ionized solid at $T=0$.

Ionic model defined by Eq. (5) corresponds to the first (Einstein) approximation for the Helmholtz free energy of a solid. It neglects not only the anharmonicity of atomic vibrations but also all correlations between displacements of pairs, triplets, etc. of atoms.

Nevertheless, as it was shown in Ref. 6, in a wide range of temperatures this approximation provides a reasonable estimation for Helmholtz free energy of a real solid. Knowing parameters of this model one may determine the equilibrium ionization degree $\bar{\xi}$ at given density and temperature by minimizing free energy with respect to $\xi$.

\section{Interparticle interaction in highly compressed hydrogen solid}

To apply the model described above, one must know at least four functions of density $\rho$ and ionization degree $\xi$ : the static potential energy $U_{N}^{\text {(st) }}$ and three elastic constants $\alpha^{(0)}, \alpha^{(+)}, \alpha^{(-)}$. The simplest way to evaluate these quantities and find the equilibrium degree of ionization $\bar{\xi}$ is their direct computation on the basis of a potential model for atom-atom, ion-atom and ion-ion interactions.

Such potential model is described below. It includes two different types of interaction: intramolecular (inside $\mathrm{H}_{2}$ and $\mathrm{H}_{2}^{(+)}$diatomics) and intermolecular (interparticle). The last one include short range (repulsion + ion-atom polarization), and long range Coulomb ion-ion interactions:

$$
U_{N}^{(\text {st })}(\rho, \xi)=U_{N}^{(\text {intra })}(\rho, \xi)+U_{N}^{\text {(short) }}(\rho, \xi)+U_{N}^{C}(\rho, \xi) .
$$

$\mathrm{N}$-particle potential energy in this work for all types of interaction was represented within atom-atom approximation (AAA) [7], i.e.:

$$
U_{N}^{(k)}=\sum_{i<j}^{N} \phi_{i j}^{(k)}\left(r_{i j}\right)
$$

where $\phi_{i j}^{(k)}(r)$ are atom-atom, atom-ion or ion-ion central interaction potentials.

Below we describe specific types of interaction included in our potential model in details. 


\subsection{Intramolecular interaction}

Potential energy $U_{N}^{\text {(intra) }}(\rho, \xi)$ includes interaction of hydrogen atoms inside neutral $\mathrm{H}_{2}$ molecules and positive ions inside $\mathrm{H}_{2}^{(+)}$molecular ions. $\mathrm{H}-\mathrm{H}$ interaction inside neutral $\mathrm{H}_{2}$ molecule was supposed to be the same ${ }^{1} \Sigma_{g}^{+}$-curve as in ideal diatomic gas.

It was represented by the modified Hulburt-Hirshfelder potential [11]:

$$
U\left({ }^{1} \Sigma \mid r\right)=D_{e}\left[\exp (-2 x)-2 \exp (-x)-a x^{3}(1-b x) \exp (-c x)\right]
$$

where $x=\beta\left(r / r_{e}-1\right)$. We adopted the following set of potential parameters for $\mathrm{H}_{2}: \beta=1.4403 ; r_{e}=0.74126 \AA$, $D_{e}=4.767 \mathrm{eV}, a=0.1156, b=1.0215, c=1.72$, which give an excellent approximation of the ${ }^{1} \Sigma_{g}^{+}$-curve within a wide range of distances $(0.3-5 \AA)$ [11].

Potential energy of $\mathrm{H}_{2}^{(+)}$molecular ion was described by the same analytical expression Eq. (8). The exact ${ }^{1} \sigma_{g}$-interaction energy curve of $\mathrm{H}_{2}^{(+)}$ion [12] was fitted by Eq. (8) and the following set of parameters obtained:

$$
\begin{gathered}
\beta=1.3558 ; r_{e}=1.0584 \AA, D_{e}=2.793 \mathrm{eV}, a=0.2803, \\
b=0.000407, c=5.37 .
\end{gathered}
$$

\subsection{Short-range repulsion}

Potential energy of short-range repulsion $U_{N}^{\text {(short) }}(\rho, \xi)$ includes contributions from all pairs of atoms and ions, both free or bonded in different diatomics. $\mathrm{H}_{2}-\mathrm{H}_{2}$ interaction energy within AAA [7] is the sum of four central atom-atom potentials $\phi_{\mathrm{H}-\mathrm{H}}(r)$ defined as

$$
\phi_{\mathrm{H}-\mathrm{H}}(r)=\frac{1}{4} U\left({ }^{1} \Sigma \mid r\right)+\frac{3}{4} U\left({ }^{3} \Sigma \mid r\right) .
$$

Here $U\left({ }^{1} \Sigma \mid r\right)$ and $U\left({ }^{3} \Sigma \mid r\right)$ are potential energies of two hydrogen atoms in their singlet ${ }^{1} \Sigma_{g}^{+}$and triplet ${ }^{3} \Sigma_{u}^{-}$states. We used the analytical representation of the atom-atom potential Eq. (9) proposed by Saumon and Shabrier [8]:

$\phi_{\mathrm{H}-\mathrm{H}}(r)=\varepsilon\left\{\gamma \exp \left[-2 s_{1}\left(r-r^{*}\right)\right]-(1+\gamma) \exp \left[-s_{2}\left(r-r^{*}\right)\right]\right\}$.

Five parameters of this potential: $r^{*}=3.2809 \AA, \varepsilon=$ $=1.74 \cdot 10^{-3} \mathrm{eV}, \quad \gamma=0.4615, \quad s_{1}=1.6367 \AA^{-1}, \quad s_{2}=$ $=1.2041 \AA^{-1}$ have been determined in Ref. 8 .

At moderate compressions this set of parameters describes the equation of state [7], as well as the melting and orientation phase transitions [9]. At higher compressions the interatomic distances become too short and differences between predicted and measured pressures (see Ref. 10 and references therein) become essential. Potential Eq. (10) with above parameters overestimates repulsion of hydrogen atoms at short distances.

We performed re-calibration of the atom-atom potential for $\mathrm{H}-\mathrm{H}$ interaction by fitting two of its parameters: $r^{*}$ and $s_{1}$. The resulting values $r^{*}=4.527 \AA ; s_{1}=0.9 \AA^{-1}$ provide an excellent fit of the pressure-volume relation for molecular hydrogen calculated in Ref. 10 in volumes range from 2.0 to $8.0 \mathrm{~cm}^{3} / \mathrm{mol}$.

For all other short-range repulsive interactions we adopted the following simple analytical form, which reflects the extremely soft repulsion in hydrogen:

$$
\phi_{i j}^{(\text {rep })}(r)=\frac{A_{i j}}{r^{5}} .
$$

Parameter $A_{i j}$, in general, must be different for different types of interactions (neutral atom-ion and ion-ion). Below we will discuss the problem of determination of this parameter for different types of interaction.

\subsection{Ion-atom polarization potential}

An important contribution to the short-range ion-atom interaction is polarization of surrounding hydrogen molecules. Its inclusion makes the ionic model more realistic in prediction of stability conditions in partly ionized hydrogen solid. Contrariwise, inclusion of mutual polarization of ions is less important, because it is only a small portion of their strong Coulombic interaction. Therefore in this work we treat ions as nonpolarizable particles.

Additionally, we ignore within current potential model the difference between polarizability of free $\mathrm{H}$ atom and atom in $\mathrm{H}_{2}$ molecule and estimate the potential energy of ion-atom polarization interaction at long distances by using the standard asymptotic form:

$$
\phi_{i j}^{(\mathrm{pol})}(r)=-\frac{e^{2}|Z| \alpha}{2 r_{i j}^{4}},
$$

where $\alpha$ is polarizability of free hydrogen atom ( $\alpha=4.5$ a.u.), $r_{i j}$ is the distance between $i$ th atom and $j$ th ion and $Z$ is its formal charge $(Z=-1$ for the negative ion and $Z=0.5$ for the positive one) [13].

\subsection{Short-range ion-atom interaction potential}

At high densities the distances between ions and surrounding neutral atoms become too short to apply Eq. (12) without any correction. Therefore we introduce a more general form of the ion-atom interaction potential, which includes both short-range repulsion Eq. (11) and polarization contributions Eq. (12):

$$
\phi_{i j}^{(\text {short })}(r)=\frac{e^{2}|Z| \alpha}{2 R_{i}^{4}}\left[\left(\frac{R_{i j}}{r}\right)^{5}-\left(\frac{R_{i j}}{r}\right)^{4}\right] .
$$

Here $R_{i j}$ is the radius (characteristic size) of ion-atomic interaction (at $r=R_{i j}$ the short-range repulsion compensates the polarization attraction, i.e., $\phi_{i j}^{\text {(short) }}\left(R_{i j}\right)=0$ ). At the long distances Eq. (13) tends to Eq. (12) and at small distances approaches to Eq. (11). 


\subsection{Long-range interaction}

The last (but not least) contribution to the ionic interaction is the intensive and long-range Coulombic part $U_{N}^{(C)}(\rho, \xi)$. It is the most important type of interionic interaction. This contribution was taken into account according to the method of effective (pre-averaged) Ewald interionic potentials proposed earlier [14]

$$
U_{N}^{(C)}=-\sum_{i=1}^{N} \frac{3 e^{2} Z_{i}^{2}}{16 \pi \varepsilon_{0} r_{m}}+\frac{1}{2} \sum_{i=1}^{N} \sum_{j=1, j \neq i}^{N} \tilde{\phi}\left(r_{i j}\right) .
$$

Here $\tilde{\phi} r_{i j}$ is the effective ion-ion potential defined as

$$
\tilde{\phi}\left(r_{i j}\right)= \begin{cases}\frac{e^{2} Z_{i} Z_{j}}{4 \pi \varepsilon_{0} r}\left\{1+\frac{1}{2}\left(\frac{r}{r_{m}}\right)\left[\left(\frac{r}{r_{m}}\right)^{2}-3\right]\right\}, & r<r_{m}, \\ 0, & r>r_{m} .\end{cases}
$$

$Q_{i}$ are charges of ions, and $r_{m}$ is the radius of sphere having the same volume as the computer simulation cell.

This method was proved to be an effective tool for simulation of disordered ionic solids and was used in our simulations before $[15,16]$ (see Refs. $14-16$ for details).

\section{Properties of conducting phase from computer simulation}

Molecular dynamics technique, analogous to that used earlier in our study of pre-melting and melting phase transitions in ionic solids [16], when combined with the potential model described in the previous Section was applied to calculate pressure, energy, and electrical conductivity of partly ionic solid hydrogen directly.

\subsection{General scheme of computation procedure}

The adopted computation procedure includes four stages.

1. Minimization of the static potential energy with respect to positions of all atoms and ions in simulation cell at given density $\rho$ and ionization degree $\xi$ (at $T \rightarrow 0$ ), i.e., computation of $U_{N}^{(\mathrm{st})}(\rho, \xi)$; and estimation of elastic constants $\alpha^{(0)}, \alpha^{(+)}, \alpha^{(-)}$by sequential displacing of all atoms and ions in the cell.

2. Determination of equilibrium ionization degree $\bar{\xi}$ by minimizing Helmholtz free energy Eq. (5) at given density and temperature in partly ionic phase.

3. Estimation of the phase transition line (i.e., transition pressure and densities of co-existing phases at given temperature) using Eq. (5).

4. Evaluation of electrical conductivity in molecular dynamics simulation at given density, temperature and equilibrium ionization degree $\bar{\xi}$.

\subsection{Calibration of the model}

Of course, any assessment based on the ionic model depends on the adopted parameters of the potential model and of the size of simulation cell. In general, radii $R_{i j}$ in

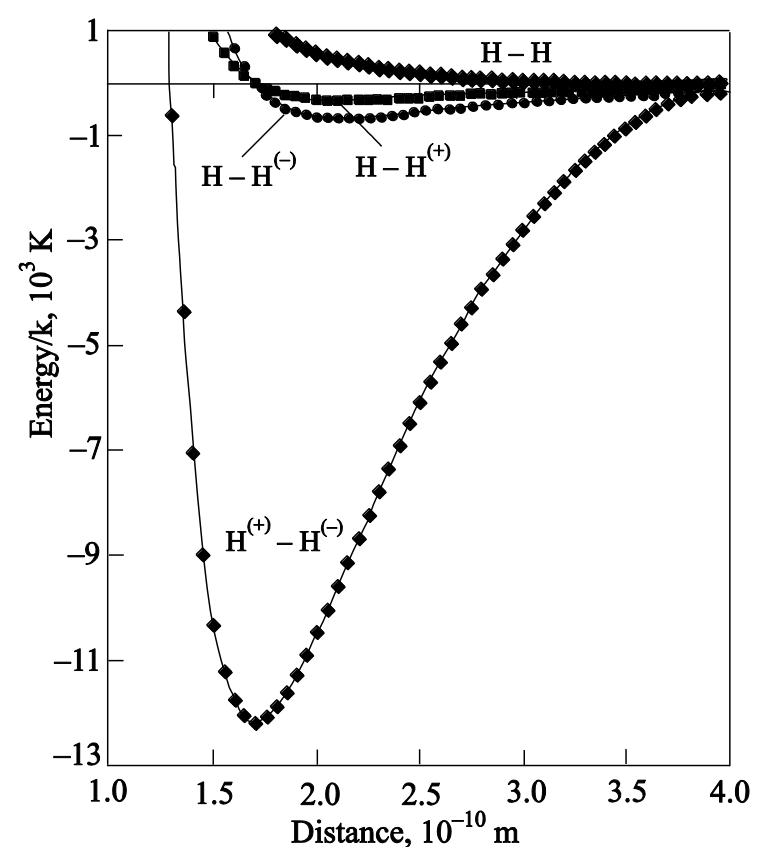

Fig. 1. Interaction potentials of atoms and ions of hydrogen. Minimum of $\mathrm{H}^{(+)}-\mathrm{H}^{(-)}$curve and zeroes of $\mathrm{H}-\mathrm{H}^{(-)}$and $\mathrm{H}-\mathrm{H}^{(+)}$curves correspond to the adopted ionic radius $R_{i}=1.68 \AA$.

Eq. (13) for positive and negative ions as well as repulsion parameters $A_{i j}$ in Eq. (11) for ion-atom and ion-ion interactions are different. However, in this work we adopted the simplest possible scheme, which has only one free parameter for all ions and all interactions.

We used the standard definition of ionic radius $R_{I}$ as a distance, where Coulomb forces compensate the shortrange attraction of positive $\mathrm{H}^{(+)}$and negative $\mathrm{H}^{(-)}$ions. Parameters $R_{i j}$ for $\mathrm{H}-\mathrm{H}^{(-)}$and $\mathrm{H}-\mathrm{H}^{(+)}$curves in Eq. (13) were set equal to $R_{I}$.

Repeating the above stages 1-3 with different values of this effective radius, we adopted finally the value $R_{j}=$ $=1.68 \AA$ which allow reproducing the observed pressure of transition to the conducting phase at room temperature (230 GPa) [4]. The corresponding interaction potentials of atoms and ions are shown in Fig. 1. Below we discuss the details of above calculations and some results obtained during simulations.

\subsection{Equilibrium ionization degree}

The dependence of the excess Helmholtz free energy, i.e., the difference between absolute Helmholtz free energy of partly ionized and molecular hydrogen: $\Delta F(T, \rho, \xi)=$ $=F(T, \rho, \xi)-F(T, \rho, 0)$, from ionization degree $\xi$ at room temperature and different densities is presented in Fig. 2.

As one can see, at the relatively low density $\left(\rho<1.0 \mathrm{~g} / \mathrm{cm}^{3}\right)$ this dependence is monotonous. The excess free energy increases with increase of number of ions because the sum of polarization energy and Coulomb attraction cannot compensate the ionization energy. Molecular solid remains the only stable state here. At higher 


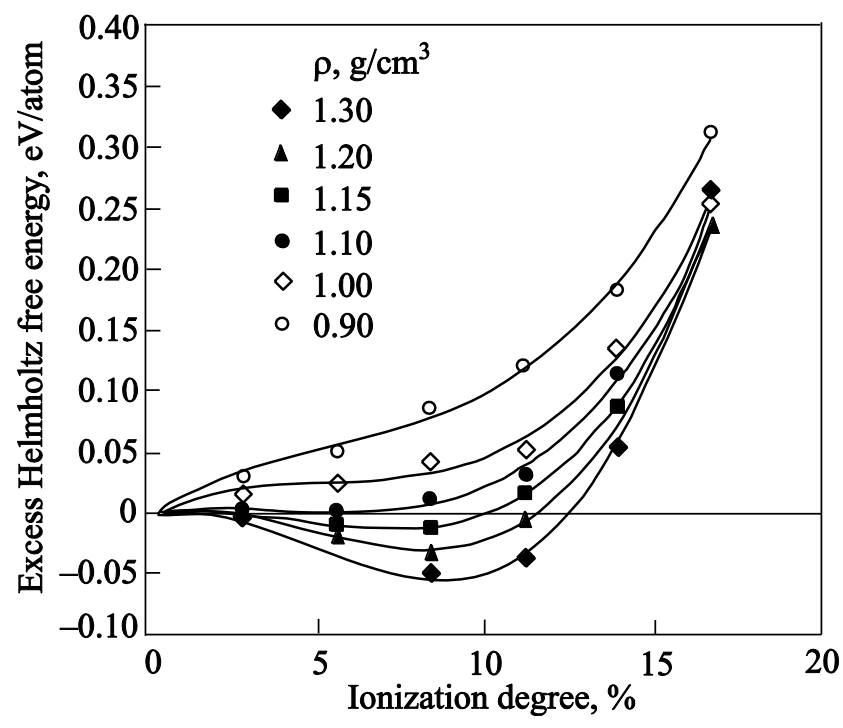

Fig. 2. Excess Helmholtz free energy (per atom) of partly ionized solid hydrogen as a function of ionization degree at different densities (shown in legend in $\mathrm{g} \cdot \mathrm{cm}^{-3}$ ).

density the initial (positive) slope of this curve (which is determined by the difference between ionization and polarization energies) decreases. At a certain degree of ionization the Coulombic contribution overcomes this tendency: the excess free energy reaches maximum and began decrease with the increase of ionization degree. This decrease then slows down; free energy reaches a minimum and then again increases due to increasing role of the short-range repulsion forces.

As a result, at the $\Delta F(\xi)$-curve appear two extreme: a maximum at lower ionization degree and a minimum at higher ionization degree. The equilibrium degree of ionization $\bar{\xi}$ at given temperature and density was determined as the abscissa of this minimum. At certain conditions these minima may correspond to thermodynamically stable states of partly ionized hydrogen solid. Locations of these minima are plotted in Fig. 3 as functions of pressure at two temperatures $(T=300 \mathrm{~K}$ and $T=3000 \mathrm{~K})$. Open symbols correspond to metastable states of partly ionized hydrogen solid and full symbols to stable states (details of the phase equilibria calculation are explained in the next subsection). The maximum degree of ionization, which was estimated in this way, is slightly increasing with pressure but remains small: about $8 \%$ at room temperature and about $10 \%$ at $T=3000 \mathrm{~K}$.

\subsection{Phase transition line}

After determination of the equilibrium ionization degree $\bar{\xi}(T, \rho)$, the transition pressure $P_{t}$ and densities of coexisting phases were estimated using a standard method of double tangent. Helmholtz free energy of molecular hydrogen $F(T, \rho, 0)$ and of the partly ionic state $F(T, \rho, \bar{\xi}(T, \rho))$ were plotted at fixed temperature against molar volume $\left(V=\rho^{-1}\right)$, and the transition pressure $P_{t}$ was determined as a slope of their common tangent. At

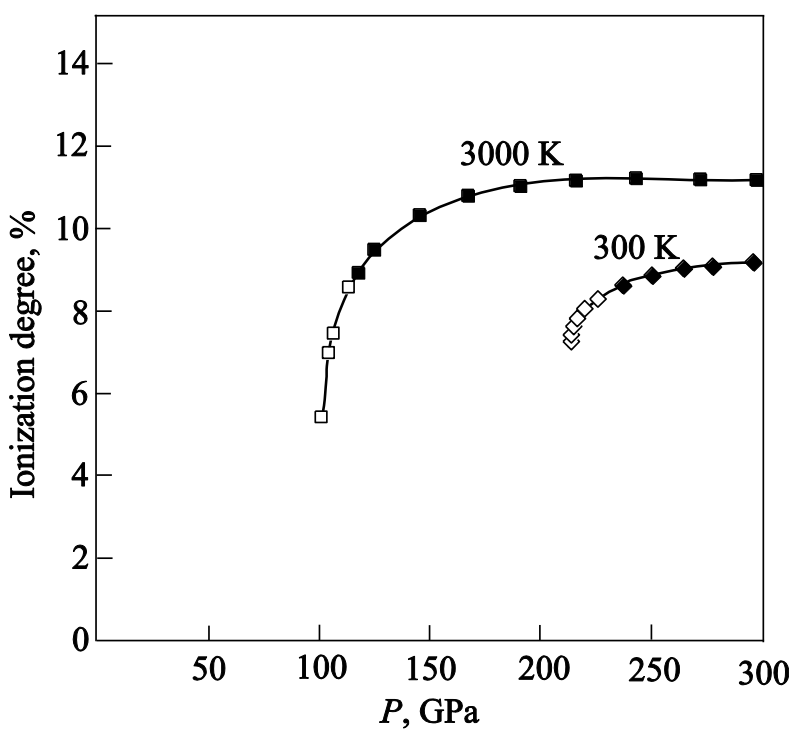

Fig. 3. Equilibrium ionization degree as a function of pressure. Open symbols correspond to metastable and full symbols to stable states at $T=300 \mathrm{~K}, T=3000 \mathrm{~K}$.

$P_{t}=230 \mathrm{GPa}$ and room temperature the volume change $\Delta V$ was found to be relatively small $\left(0.065 \mathrm{~cm}^{3} / \mathrm{mol}\right)$. With increasing temperature the transition pressure decreases and the volume change increases $\left(P_{t}=110 \mathrm{GPa}\right.$ and $\Delta V \approx 0.3 \mathrm{~cm}^{3} / \mathrm{mol}$ at $\left.3000 \mathrm{~K}\right)$. This is illustrated in Fig. 4. The estimated location of the transition line from molecular (phase III) into conducting phase (IV) is shown by dashed line in Fig. 5.

\subsection{Estimation of electrical conductivity}

Molecular dynamics technique provides an easy way to determine conductivity of ionic systems by applying an

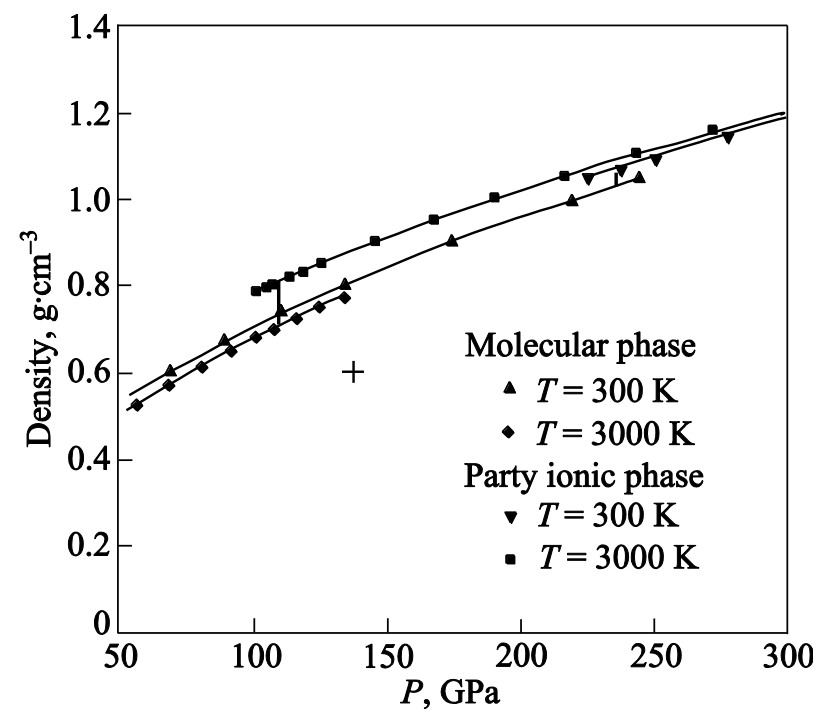

Fig. 4. Pressure dependence of density in compressed solid hydrogen at two temperatures (300 and $3000 \mathrm{~K}$ ). Vertical line segments correspond to locations of phase transition. Cross indicates the state in which electrical conductivity was observed in shockcompression experiments in liquid phase [3]. 


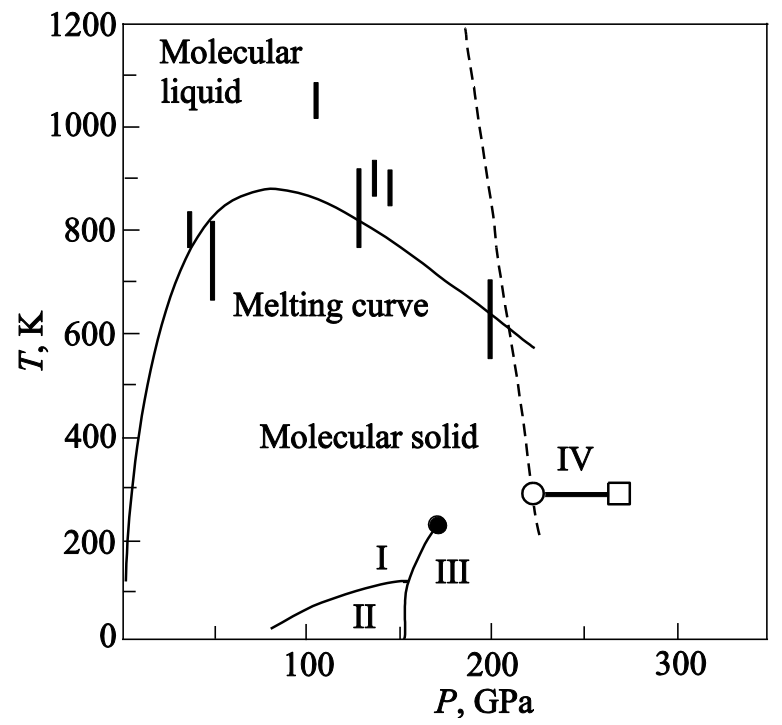

Fig. 5. Phase diagram of compressed hydrogen. Open circle shows the transition from phase III to phase IV and open square the next transition discovered by Eremets and Troyan [4] at $T=$ $=300 \mathrm{~K}$. Vertical bars indicate probable location of melting line predicted ab initio [19]. Dashed line shows the location of the transition line from molecular solid phase III to the partly ionic solid phase IV estimated in this work.

external electric field and monitoring numbers of positive and negative charges entering and leaving the cell.

According to estimations made by Nellis, Ruoff and Silvera [2], conductivity in phase IV discovered by Eremets and Troyan [4] is very low (about $10^{-2} \Omega^{-1} \mathrm{~m}^{-1}$ ). Unfortunately, in our computer simulation (we used a rather small cell containing only 216 hydrogen atoms) one cannot determine the corresponding charge flux in acceptable simulation time using the same field strength as in experiments of Eremets and Troyan [4]. Therefore we were forced to apply external field two or three orders of magnitude higher to get results of reasonable significance level in acceptable computer time.

Figure 6 illustrates the results of such simulations at room temperature and two pressures. In both cases the estimated average slope of the logarithm of electrical conductivity in the Arrhenius plot was small and negative. The predicted temperature dependence of electrical conductivity is in a good agreement with experimental data. According to measurements of Eremets and Troyan [4], the activation energy in conducting phase of solid hydrogen is about $8 \mathrm{meV}$ while our estimations are $7.4 \mathrm{meV}$ at $250 \mathrm{GPa}$ and $8.0 \mathrm{meV}$ at $270 \mathrm{GPa}$. The predicted pressure dependence of resistivity was also negative like in DAC experiments [4] but is much less pronounced. At the same time the predicted absolute values of conductivity are much higher then in experiment [4]. By decreasing external field we found a strong decrease of estimated conductivity. This gives some hope for the agreement between the results of modeling and expe-

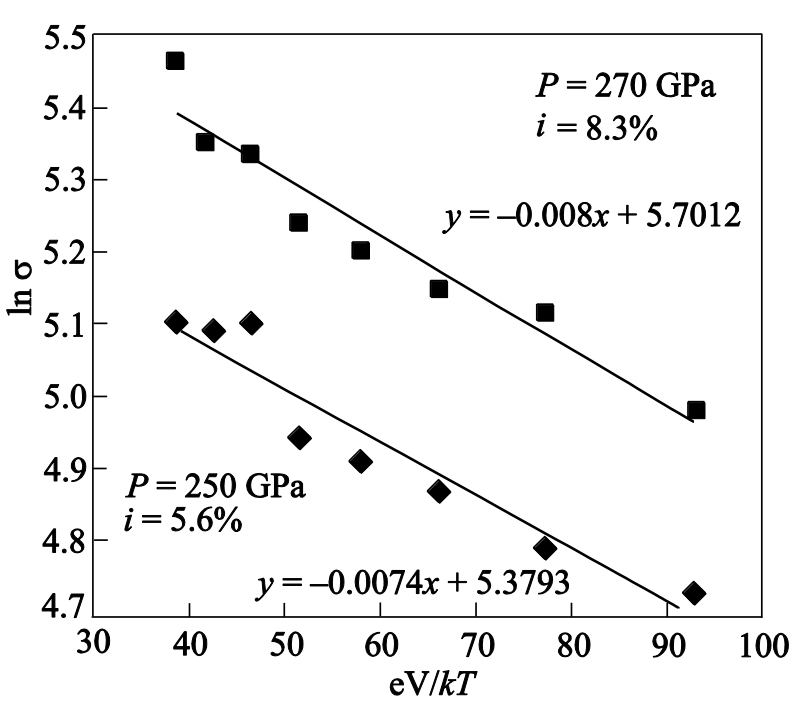

Fig. 6. Temperature dependence of electrical conductivity (in $\Omega^{-1} \cdot \mathrm{m}^{-1}$ ) of partly ionic hydrogen solid estimated at two pressures.

riment in weaker fields that are not available now for us to simulate.

\section{Conclusions}

Recent DAC experiments of Eremets and Troyan at room temperature and megabar pressures [4] reveal transition of solid hydrogen to a nonmetallic phase IV with a low and rising with temperature conductivity. To explain such behavior of compressed hydrogen solid we propose in this work a simple ionic model.

This model accounts for dissociative ionization of hydrogen molecules into stable positive molecular $\mathrm{H}_{2}^{(+)}$and negative atomic $\mathrm{H}^{(-)}$ions. The model proposed has only one parameter - ionic radius, which was fitted to reproduce the pressure of transition to the conducting phase at room temperature [4]. We estimated the equilibrium ionization degree in partly ionic conducting state by minimizing Helmholtz free energy, transition pressure and volume change and evaluated electrical conductivity by molecular dynamics technique.

The main conclusion, which can be made on the basis of our calculations, is that the ionic migration mechanism can explain some characteristics of the compressed conducting hydrogen. Ionic model reproduces the negative temperature dependence of resistivity, observed in static DAC experiment [4] at room temperature. Our estimations are also in line with results of dynamic experiments on multiply shocked hydrogen at $T=3000 \mathrm{~K}$ [3]. We found a reasonable agreement between the predicted pressure of transition at $3000 \mathrm{~K}(110 \mathrm{GPa})$, and parameters of conducting state observed in the fluid phase (140 GPa and $\left.0.6 \mathrm{~g} / \mathrm{cm}^{3}\right)$ in shock compression experiments [3].

Correctness of assumptions used in this work can be verified experimentally. First of all, the presence of negative 
hydrogen ions, even in a small amount, can be detected in optical spectra of compressed hydrogen. The estimated vibronic frequency of $\mathrm{H}_{2}^{(+)}$is about half as much as vibronic frequency of $\mathrm{H}_{2}$ and may be obscured by the wellknown lattice absorption band of diamond [18]. This is a challenge for experimentalists.

Secondly, the fact of the volume change $\Delta V$ increase with increasing temperature can be verified. Such behavior, if confirmed by experiment, may be related to existence of a lower critical point on the transition line. This, in turn, may answer the question why so many sophisticated DAC experiments (see discussion in Ref. 2 and references therein) do not reveal any conductivity in solid hydrogen at cryogenic temperatures and megabar pressures.

Of course, the model proposed in this work is actually only a basic one, developed to explain only the principal features of solid hydrogen in conducting phase. It does not take into account many aspects which may be important in a wider context. It ignores the difference in effective radii of positive and negative ions, as well as contribution of quantum effects, anharmonicity and correlations, as well as contribution of other mechanisms of electric charge transport, like polaron hopping etc. In this regard it should be noted that very high electric fields applied in our simulations make partly ionic hydrogen solid close to the electric breakdown and the mechanism of conductivity observed in experiments [4] may differ from that under simulation conditions. We must note also other attempts to explain unusual behavior of phase IV [17].

An unanswered question remains also the possibility to reconcile ionic model with $a b$ initio simulations [19]. It seems not clear yet how to interpret the results of ab initio calculations of in terms of the ionic model.
1. E. Wigner and H.B. Huntington, J. Chem. Phys. 3, 764 (1935).

2. W.J. Nellis, A.L. Ruoff, and I.F. Silvera, e-print arXiv:1201.0407 (2012).

3. S.T. Weir, A.C. Mitchell, and W.J. Nellis, Phys. Rev. Lett. 76, 1860 (1996).

4. M.I. Eremets and I.A. Troyan, Nature Mater. 10, 927 (2011).

5. L.V. Gurvich and I.V. Veyts, Thermodynamic Properties of Individual Substances: Elements and Compounds, Hemisphere (1990).

6. L.N. Yakub and E.S. Yakub, J. Chem. Phys. 136, 144508 (2012).

7. E.S. Yakub, Int. J. Thermophys. 22, 505 (2001).

8. D. Saumon and G. Chabrier, Phys. Rev. A 44, 5122 (1990).

9. E.S. Yakub, J. Low Temp. Phys. 122, 559 (2001).

10. Yu.A. Freiman, A. Grechnev, S.M. Tretyak, A.F. Goncharov, and R.J. Hemley, Phys. Rev. B 86, 014111 (2012).

11. E.S. Yakub, High Temp. 33, 93 (1995).

12. J.C. Slater, Quantum Theory of Molecules and Solids: Electronic Structure of Molecules. McGraw-Hill., NY (1963).

13. P. Schwerdtfeger, Atomic Static Dipole Polarizabilities, in: Atoms, Molecules and Clusters in Electric Fields: Theoretical Approaches to the Calculation Electric Polarizability, G. Maroulis (ed.), Imperial College Press (2006).

14. E. Yakub and C. Ronchi, J. Chem. Phys. 119, 11556 (2003).

15. E. Yakub, J. Phys. A: Math. Gen. 39, 4643 (2006).

16. E. Yakub and C. Ronchi, J. Chem. Phys. 127, 094508 (2007).

17. C.J. Pickard, M. Martinez-Canales, and R.J. Needs, Phys. Rev. B 85, 214114 (2012); ibid. B86, 059902 (2012).

18. J. Walker, Rep. Prog. Phys. 42, 108 (1979).

19. I. Tamblyn and S.A. Bonev, Phys. Rev. Lett. 104, 065702 (2010). 